\title{
Evaluation of the pathophysiology of classical trigeminal neuralgia by blink reflex study and current perception threshold testing
}

\author{
Ming Feng Liao $\cdot$ Meng Lee $\cdot$ Mei Jen Hsieh • \\ Mei Yun Cheng · Jiann Der Lee · Hsu Huei Weng • \\ Long Sun Ro
}

Received: 30 November 2009/ Accepted: 2 February 2010/Published online: 26 February 2010

(C) Springer-Verlag 2010

\begin{abstract}
We recruited 49 patients with classical trigeminal neuralgia (TN) according to the latest guidelines of the International Classification of Headache Disorders, and divided them into an acute ( $\leq 30$ days onset; 13 patients) and a chronic ( $>30$ days onset; 36 patients) group. We used blink reflex study and current perception threshold (CPT) testing to evaluate the painful facial areas and contralateral non-painful areas of patients with classical TN. CPT $5 \mathrm{~Hz}$ examinations, which correlate with unmyelinated fiber function, showed significantly decreased CPTs in the acute stage $(11.62 \pm 6.99$ vs. $18.69 \pm 9.66$, $P=0.025)$, but significantly increased CPTs in the chronic stage $(26.67 \pm 18.65$ vs. $19.69 \pm 13.70, P=0.010)$ on the painful side when compared with the contralateral nonpainful side. However, CPTs at $250 \mathrm{~Hz}(\mathrm{~A} \delta)$ and $2000 \mathrm{~Hz}$
\end{abstract}

M. F. Liao and M. Lee contributed equally to the study.

M. F. Liao · M. Y. Cheng · L. S. Ro ( $\square)$

Department of Neurology, Linkou Headquarters,

Chang Gung Memorial Hospital and College of Medicine,

Chang-Gung University, 199, Tung Hwa North Road,

Taipei, Taiwan

e-mail: cgrols@adm.cgmh.org.tw

M. Lee · J. D. Lee

Department of Neurology, Chiayi Branch,

Chang Gung Memorial Hospital and College of Medicine,

Chang-Gung University, Taipei, Taiwan

M. J. Hsieh

Department of Neurology, Kaohsiung Branch,

Chang Gung Memorial Hospital and College of Medicine,

Chang-Gung University, Taipei, Taiwan

H. H. Weng

Diagnostic Radiology, Chiayi Branch,

Chang Gung Memorial Hospital and College of Medicine,

Chang-Gung University, Taipei, Taiwan
(A $\beta$ ) examinations did not show significant differences between the painful and non-painful sides. In contrast, only three (3/49) patients showed an abnormal trigeminal nerve stimulation on the ipsilateral painful side by blink reflex study. The findings suggest that classical TN is not a simple large-myelinated nerve fiber dysfunction but a more complex process with a main dysfunction of unmyelinated nerve fibers.

Keywords Current perception threshold .

Classical trigeminal neuralgia - Unmyelinated fiber .

Neuropathic pain

\section{Introduction}

Classical trigeminal neuralgia (TN) has an annual incidence rate of 4.5 per 100000 [1]. It is characterized by recurrent episodes of intense, lancinating pain evoked by light touch localized to a small area of the face. Persistent background dull pain is also noted in some chronic cases [2-4]. Most classical TN is caused by the compression of the trigeminal nerve root by tortuous or aberrant vessels within a few millimeters of entry into the pons [5].

Current opinions indicate that pain in classical TN is caused by primary demyelination damage to large rather than small afferent fibers [5-7]. Different neurophysiologic methods have been used in TN investigations but with inconclusive results. Few studies of quantitative sensory testing showed that the cutaneous perception of temperature and light touch is impaired within the affected trigeminal divisions $[8,9]$, suggesting both small and large diameter sensory fiber involvement. A previous electrophysiological study showed that the trigeminal-evoked potential is abnormal in $\mathrm{TN}$ patients, indicating a large 
myelination fiber dysfunction [10]. However, only few patients with idiopathic TN have an abnormal blink reflex $[11,12]$, which is in conflict with large myelination fiber impairment. There were also reports about using laserevoked potentials, which resulted in a small myelinated fiber dysfunction in TN [13-15]. A recent study using nociceptive blinking reflex (nBR) and pain-related-evoked potential (PREP) showed a dysfunction of the trigeminal nociceptive pathway caused by small-myelinated A-delta fiber dysfunction [16]. The above studies had inconsistent results, and which types of nerve fibers are most affected is not well clarified yet.

Although the ordinary-evoked potential, blink reflex and laser-evoked potential all can be used to detect electrophysiological abnormalities in TN [10-15], they only offer information on large myelinated fibers and small myelinated fibers, respectively. Small unmyelinated fibers cannot be evaluated. Constant current perception threshold (CPT) testing is another method, which can evaluate both largeand small-fiber functions at the same time [17-19]. CPT relates to the direct stimulation of myelinated and unmyelinated nerve fibers in the skin. The $2000 \mathrm{~Hz}$ stimulusevoked responses from large myelinated fibers $(\mathrm{A} \beta)$, $250 \mathrm{~Hz}$ stimulated small myelinated fibers $(\mathrm{A} \delta)$, and $5 \mathrm{~Hz}$ induced responses from unmyelinated fibers [17-19]. CPT has been widely used in the assessment of diabetic neuropathy [20], uremic neuropathy [21], post-therapeutic neuralgia [22] and Fabry's disease [23].

The purpose of this study aims to clarify which nerve fibers are mainly affected in classical TN by blink reflex and CPT studies. We will also differentiate acute and chronic pain according to different consensus [2, 24-26] and also try to study if there is any electrophysiological change between the acute and chronic stage.

\section{Patients and methods}

\section{Clinical evaluation}

Patients with paroxysmal attacks of pain, affecting one or more divisions of the trigeminal nerve were recruited. Patients with no clinically evident neurological deficit by image studies including CT or MRI were defined as classical TN according to the latest guidelines of the International Classification of Headache Disorders [2]. We recruited 49 patients in this study. All patients were evaluated by detailed history taking, neurological examinations, and CPT testing and all patients were evaluated by brain computed tomography (CT) or magnetic resonance imaging (MRI) studies (39 and 10 patients, respectively) to rule out symptomatic TN. All patients also had blink reflex study. The durations between the symptoms onset and the time of receiving examinations are recorded. We tried to set the cut off points at 1,3 , and 6 months to differentiate acute from chronic pain according to the definition of chronic pain by different consensus [2, 24-26]. This study was approved by the Institutional Review Board of Chang Gung Memorial Hospital and University.

Blink reflex studies

Surface electrodes were used to stimulate the nerve and record the evoked muscle action potentials [27]. The recording and reference electrodes were placed on the lower aspect of the orbicularis oculi muscle with a ground electrode over the forehead. A constant-current unit was used to stimulate the supraorbital nerve. The reflex responses from the orbicularis oculi muscle were then simultaneously recorded on both sides.

\section{CPT testing}

All patients were required to cease medications including anticonvulsants, tricyclic antidepressants, serotonin-norepinephrine reuptake inhibitors, opioid analgesics, topical lidocaine patches or gels and non-steroidal anti-inflammatory drugs (NSAIDs) for 3 days before receiving CPT testing. The methodology was similar to the one described previously [17]. The Neurometer (Neurotron Inc., Baltimore, MD) was used. The Neurometer is a constant-current stimulator capable of delivering stimuli at three different frequencies. Each patient was tested at three sinusoidal stimulation frequencies $(2000,250,5 \mathrm{~Hz})$ within the painful facial area and the contralateral mirror image area. The current was applied to the face skin via a pair of $1 \mathrm{~cm}$ diameter standard gold-plated electrodes. CTPs defined $0.01 \mathrm{~mA}$ as " 1 " and $10 \mathrm{~mA}$ as "1000". At each frequency $(2000,250,5 \mathrm{~Hz})$, the current was slowly increased from 0.01 to $9.99 \mathrm{~mA}$ until the patients reported sensation. The stimulus then was turned off and lowered by $0.1 \mathrm{~mA}$, and then turned on again. This procedure was repeated until a ranged of $0.1 \mathrm{~mA}$ was established. Double blind methodology then was used to present the patient with 6-20 cycles of randomly selected real and false stimulus until the exact CPT value could be determined within $a \pm 20 \mu \mathrm{A}$ range $[22,23]$.

\section{Statistical analysis}

Continuous variables were expressed as mean \pm SD and compared by performing the paired or unpaired $t$ test. Paired $t$ tests were used to compare the CPT values between painful and contralateral non-painful sites of the same patient with classical TN. In addition, unpaired $t$ tests were used to compare the CPT values on the painful side 
between acute and chronic stages of different patients with classical TN. The level of significance was 0.05. All $P$ values were two sided. Statistical analyses were performed with a statistical software package (SPSS for Window 15.0; SPSS Inc.).

\section{Results}

After statistical analyses, we found that there was a trend that the values of $5 \mathrm{~Hz}$ CPT on the painful site were lower (hypersensitive) than the non-painful site within the first month, but they became higher (hyposensitive) than those on the non-painful site after 1 month (Fig. 1) If we set the cutoff point at 1 month to differentiate acute from chronic pain, there will be a statistically significant result. We identified 13 patients with acute classical TN with symptoms onset $\leqq 30$ days and 36 patients with chronic classical TN with symptoms onset $>30$ days. All patients evaluated by brain CT or MRI showed a normal finding. The mean durations were $10.85 \pm 10.03$ days in the acute classical TN and $21.74 \pm 27.76$ months in the chronic classical TN. The mean ages were $54.38 \pm 13.66$ and $57.23 \pm 12.79$ years for the acute and chronic groups, respectively. Overall, there were 85 and $66 \%$ females with acute and chronic classical $\mathrm{TN}$, respectively. The age and gender ratios were not significantly different in patients with acute and chronic classical TN (Table 1).

At the $5 \mathrm{~Hz}$ examinations, the values of CPTs on the painful side were significantly lower in the acute stage

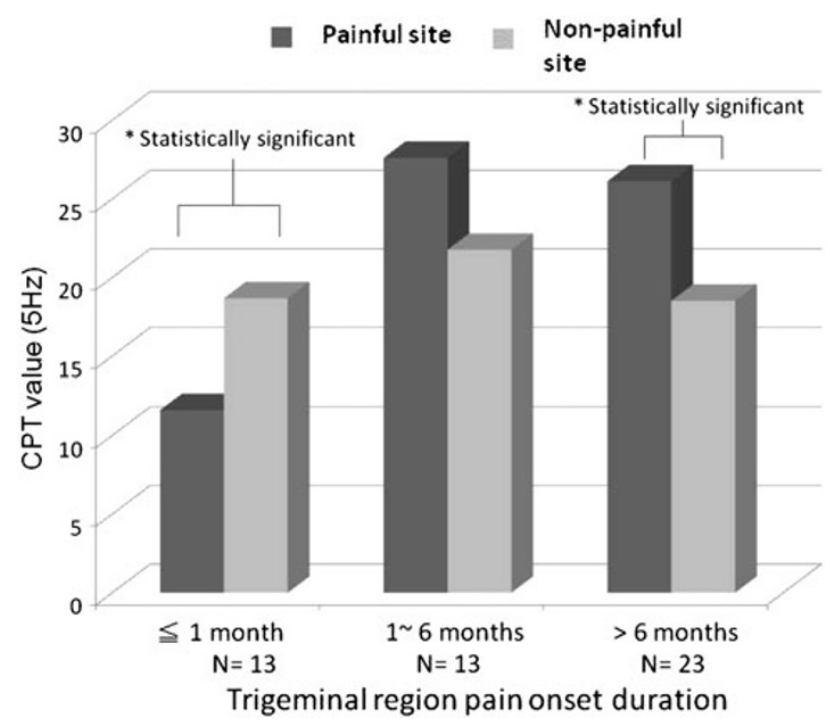

Fig. 1 Comparisons of the values of $5 \mathrm{~Hz}$ CPT between painful and non-painful sides at different times. There is a trend that the values of $5 \mathrm{~Hz}$ CPT on the painful side are lower than the non-painful side within the first month, but they evolved to be higher than the nonpainful side after one month. $(* P<0.05)$
$(11.62 \pm 6.99$ vs. $18.69 \pm 9.66, P=0.025)$, but they became significantly higher $(26.67 \pm 18.65$ vs. $19.69 \pm$ $13.70, P=0.010$ ) than those on the contralateral side in the chronic stage. At the $250 \mathrm{~Hz}$ examinations, the values of CPTs were not significantly different between the painful and non-painful sides in patients with acute $(30.23 \pm 12.29$ vs. $38.00 \pm 14.62, P=0.168)$ and chronic classical TN $(41.00 \pm 22.17$ vs. $36.42 \pm 16.34, \quad P=$ 0.179). Also at the $2000 \mathrm{~Hz}$ examinations, the values of CPTs were not significantly different between the painful and non-painful sides in patients with acute (101.23 \pm 40.11 vs. $122.77 \pm 31.89, P=0.066)$ and chronic classical TN (125.67 \pm 43.23 vs. $124.58 \pm 45.83, P=0.851)$ (Table 2).

At the $5 \mathrm{~Hz}$ examinations, the values of CPTs were significantly higher in the chronic stage than in the acute stage on the painful side of classical TN (26.67 \pm 18.65 vs. $11.62 \pm 6.99, P=0.000)$. However, there were no significant differences on the painful side between the acute and chronic stage at the 250 and $2000 \mathrm{~Hz}$ examinations, respectively $(30.23 \pm 12.29$ vs. $41.00 \pm 22.17, P=0.105$, $101.23 \pm 40.11$ vs. $125.67 \pm 43.23, P=0.082$ ) (Table 3 ).

All patients also received a blink reflex study and only three patients showed an abnormal finding by this study. All of the three patients showed prolonged ipsilateral R1 and bilateral R2 potentials after stimulation of the ipsilateral trigeminal nerve on the painful sides. One patient was in an acute stage and the other two were in a chronic stage. Although blink reflex study relates to large-myelinated nerve fiber dysfunction [27], these three patients did not show abnormal findings at the CPT $2000 \mathrm{~Hz}$ examinations.

\section{Discussion}

Our results show that classical $\mathrm{TN}$ is commonly found in female patients, which is similar to previous studies [1]. Our study also showed that unmyelinated nerve fiber sensation thresholds of classical TN decrease in the acute stage but increase in the chronic stage when the cut off duration is set to 1 month. We propose that the probable underlying mechanisms of classical TN is mainly a dysfunction of the unmyelinated nerve fibers, which are in a dynamic phase over times. It might increase neuronal excitability and ectopic firing in the acute stage, and then evolves to permanent nerve injuries with overwhelming injury discharges to the central nervous system and induces subsequent central sensitization in the chronic stage. This hypothesis is compatible with the clinical signs and symptoms of patients with classical TN. Patients with classical TN usually initially present with frequent brief electric shock-like pains, abrupt in onset and cessation, and sometimes gradually convert to a 
Table 1 Demographic data of classical trigeminal neuralgia

\begin{tabular}{lllr}
\hline & Acute $(n=13)$ & Chronic $(n=36)$ & $P$ \\
\hline Duration (days/months) & $10.85 \pm 10.03$ (days) & $21.74 \pm 27.76$ (months) & $<0.001$ \\
Age (years) & $54.38 \pm 13.66$ & $57.23 \pm 12.79$ & 0.603 \\
Female:male & $11: 2$ & $2: 1$ & \\
\hline
\end{tabular}

Table 2 Comparisons of the values of CPT testing at three different frequencies on the painful and non-painful sides

\begin{tabular}{llll}
\hline & Painful & Non-painful & $P$ \\
\hline $5 \mathrm{~Hz}$ & & & \\
$\quad$ Acute $(n=13)$ & $11.62 \pm 6.99$ & $18.69 \pm 9.66$ & $0.025^{*}$ \\
Chronic $(n=36)$ & $26.67 \pm 18.65$ & $19.69 \pm 13.70$ & $0.010^{*}$ \\
$250 \mathrm{~Hz}$ & & & \\
Acute $(n=13)$ & $30.23 \pm 12.29$ & $38.00 \pm 14.62$ & 0.168 \\
Chronic $(n=36)$ & $41.00 \pm 22.17$ & $36.42 \pm 16.34$ & 0.179 \\
$2000 \mathrm{~Hz}$ & & & \\
Acute $(n=13)$ & $101.23 \pm 40.11$ & $122.77 \pm 31.89$ & 0.066 \\
Chronic $(n=36)$ & $125.67 \pm 43.23$ & $124.58 \pm 45.83$ & 0.851 \\
\hline
\end{tabular}

Values are mean \pm standard deviations in $0.01 \mathrm{~mA}$

* Statistically significant

Table 3 Comparisons of the values of CPTs at three different frequencies on the painful sides between acute and chronic stage of classical trigeminal neuralgia

\begin{tabular}{lccl}
\hline & Acute $(n=13)$ & Chronic $(n=36)$ & $P$ \\
\hline $5 \mathrm{~Hz}$ & $11.62 \pm 6.99$ & $26.67 \pm 18.65$ & $0.000^{*}$ \\
$250 \mathrm{~Hz}$ & $30.23 \pm 12.29$ & $41.00 \pm 22.17$ & 0.105 \\
$2000 \mathrm{~Hz}$ & $101.23 \pm 40.11$ & $125.67 \pm 43.23$ & 0.082 \\
\hline
\end{tabular}

Values are mean \pm standard deviations in $0.01 \mathrm{~mA}$

* Statistically significant

dull background pain or numbness with less electric shocklike pains in a chronic stage $[2,3,28]$.

In contrast, there were no significant differences of sensation thresholds in both small- and large-myelinated fibers during the acute and chronic stages. These findings may suggest a less important role of small- and large-myelinated fibers in trigeminal neurologia. Blink reflex study is routinely used to diagnose $\mathrm{TN}$, which is a main study for detecting large-myelinated $(\mathrm{A} \beta)$ nerve fiber function [29], however, it did not show an adequate sensitivity in detecting trigeminal nerve dysfunction in classical TN. In our study, only 3/49 (6.1\%) patients showed an abnormal finding by blink reflex study, which is consistent with the previous studies [11,12]. Moreover, on neurological examination, patients with TN commonly had normal light touch and vibration sensory modalities, which also suggest a normal large fiber function on their trigeminal nerve distributions. Several reports using laser-evoked potentials also showed small-myelinated fiber dysfunction in TN [13-15]. A more recent study using nBR and PREP showed dysfunction of the trigeminal nociceptive pathway, which suggested that thinly myelinated A-delta fibers are more susceptible and prone to compression than Abeta fibers in the trigeminal root entry zone [16]. According to the above findings, we suggest that in addition to the traditional hypothesis of large-myelinated fiber dysfunction in $\mathrm{TN}$, it seems that small-myelinated and unmyelinated nerve fiber dysfunctions also play an important role in classical TN.

In the past, vessels compression producing large fiber demyelination and ephaptic contact between adjacent denuded axons has been widely accepted as the etiology of classical TN [5, 6]. This hypothesis is supported by clinical observations including trigeminal nerve root compression by aberrant vessels and the improvement of clinical signs/ symptoms after a decompression surgery. However, there are only few pathological studies to support the hypothesis [6, 30-32].

Our hypothesis seems to conflict with previous nerve pathological studies in classical TN [30, 31]; however, previous reports mainly focus on the pathology of largemyelinated nerve fibers under a light microscope, which is unable to detect the pathology of unmyelinated fibers. In Devor's (12 patients) and Rappaport's reports (12 patients), disruption of axon structure, reduced axon population, deand dys-myelination of large myelinated nerve fibers were found by electronic microscopy; however, they did not pay much attention to the pathological changes of unmyelinated nerve fibers [31, 33]. In contrast, there was a pathology report mainly showing unmyelinated fiber loss in a rat model of neuropathic pain under the electron microscope [34]. On the other hand, skin biopsy stained with antibody against protein gene product 9.5 (PGP 9.5) which is a pan-axonal markers may show the intra-epidermal nerve fiber density including small-myelinated nerve $(\mathrm{A} \delta$ fiber) and unmyelinated nerve (C-fiber) [35, 36], which can potentially be used to evaluate TN theoretically. Further studies of skin biopsies and detailed trigeminal nerve electron microscopic studies to evaluate small-myelinated and unmyelinated nerve fiber pathology in classical TN patients may be warranted.

The finding that unmyelinated fibers are mainly affected in classical TN can be explained well by a loose ligation animal model. One study has shown that unmyelinated fibers are more vulnerable than large myelinated fibers 
when the compressed area is restricted to a short segment $(0.5-1 \mathrm{~mm})$ [37]. In a rat animal study, increased skin blood flow and myeloperoxidase activity were found in the hind-paw distal to the ligations of the sciatic nerve; and these findings indicated that the ligation of the sciatic nerve induced an neurogenic inflammation and nociceptive Cfibers activation [38]. In primate study, Ali et al. [39] also found a reduced number of unmyelinated nerve terminals in the epidermis of the skin of the limb distal to the L6 spinal nerve ligation as compared to the contralateral limb, which showed a major C-fiber degeneration after nerve ligation. Spontaneous activity in $\mathrm{C}$-fibers is thought to be responsible for persisted burning pain and central sensitization in the dorsal horn neurons [40]. There are also studies that showed that decreased intra-epidermal C-fiber density is related to increased pain severity in HIV-associated neuropathy and diabetes patients [41, 42]. Repeated noxious stimulation to peripheral C-fiber nociceptors may generate prolonged post-stimulus sensory disturbances and a decreased threshold of the nociceptive system called central sensitization [43]. Central facilitation of trigeminal nociceptive processing with over-activation of central sensory transmission was also observed in patients with TN with chronic pain in Obermann's study [16]. These studies probably imply that the damaged C-fiber after a compression injury in the trigeminal nerve may show spontaneous activities related to frequent brief electric shock-like pain sensations in the acute stage; and long-term repeated damage may cause a permanent injury related to increase in pain thresholds of the peripheral nociceptors and central sensitization in the chronic stage.

From this study and previous reports, it seems that classical $\mathrm{TN}$ is not a simple large-myelinated fiber dysfunction, but a more complex involvement of large-, smallmyelinated and unmyelinated nerve fibers with a main dysfunction of the unmyelinated nerve fibers over times. However, this study has some limitations. First, this study only analyzes a small sample size. Secondly, this study lacks of a longitudinal series of CPT tests in these patients. Thirdly, no skin or trigeminal nerve biopsy was available in our patients to confirm small-myelinated or unmyelinated nerve fiber injuries. Understanding the underlying pathophysiology of classical TN may improve the therapy in the future. Thus, a well designed and longitudinal series of CPTs and pathological study is warranted.

\section{Conflict of interest None.}

\section{References}

1. Katusic S, Beard CM, Bergstralh E, Kurland LT (1990) Incidence and clinical features of trigeminal neuralgia, Rochester, Minnesota, 1945-1984. Ann Neurol 27:89-95
2. Headache Classification Subcommittee of the International Headache Society (2004) The international classification of headache disorders, 2nd edn. Cephalalgia 24(Suppl 1):9-160

3. Burchiel KJ, Slavin KV (2000) On the natural history of trigeminal neuralgia. Neurosurgery 46:152-154 discussion 154-155

4. Burchiel KJ (2003) A new classification for facial pain. Neurosurgery 53:1164-1166 (discussion 1166-1167)

5. Love S, Coakham HB (2001) Trigeminal neuralgia: pathology and pathogenesis. Brain 124:2347-2360

6. Devor M, Amir R, Rappaport ZH (2002) Pathophysiology of trigeminal neuralgia: the ignition hypothesis. Clin J Pain 18:4-13

7. Bowsher D (1997) Trigeminal neuralgia: an anatomically oriented review. Clin Anat 10:409-415

8. Nurmikko TJ (1991) Altered cutaneous sensation in trigeminal neuralgia. Arch Neurol 48:523-527

9. Eide PK, Rabben T (1998) Trigeminal neuropathic pain: pathophysiological mechanisms examined by quantitative assessment of abnormal pain and sensory perception. Neurosurgery 43:11031110

10. Stohr M, Petruch F, Scheglmann K (1981) Somatosensory evoked potentials following trigeminal nerve stimulation in trigeminal neuralgia. Ann Neurol 9:63-66

11. Cruccu G, Leandri M, Feliciani M, Manfredi M (1990) Idiopathic and symptomatic trigeminal pain. J Neurol Neurosurg Psychiatry 53:1034-1042

12. Cruccu G, Biasiotta A, Galeotti F, Iannetti GD, Truini A, Gronseth G (2006) Diagnostic accuracy of trigeminal reflex testing in trigeminal neuralgia. Neurology 66:139-141

13. Cruccu G, Leandri M, Iannetti GD et al (2001) Small-fiber dysfunction in trigeminal neuralgia: carbamazepine effect on laserevoked potentials. Neurology 56:1722-1726

14. Cruccu G, Galeotti F, Iannetti GD, Romaniello A, Truini A, Manfredi M (2002) Trigeminal neuralgia: update on reflex and evoked potential studies. Mov Disord 17(Suppl 2):S37-S40

15. Truini A, Galeotti F, Pennisi E, Casa F, Biasiotta A, Cruccu G (2007) Trigeminal small-fibre function assessed with contact heat evoked potentials in humans. Pain 132:102-107

16. Obermann M, Yoon MS, Ese D et al (2007) Impaired trigeminal nociceptive processing in patients with trigeminal neuralgia. Neurology 69:835-841

17. Katims JJ, Naviasky EH, Ng LK, Rendell M, Bleecker ML (1986) New screening device for assessment of peripheral neuropathy. J Occup Med 28:1219-1221

18. Liu S, Kopacz DJ, Carpenter RL (1995) Quantitative assessment of differential sensory nerve block after lidocaine spinal anesthesia. Anesthesiology 82:60-63

19. Rendell MS, Katims JJ, Richter R, Rowland F (1989) A comparison of nerve conduction velocities and current perception thresholds as correlates of clinical severity of diabetic sensory neuropathy. J Neurol Neurosurg Psychiatry 52:502-511

20. Masson EA, Veves A, Fernando D, Boulton AJ (1989) Current perception thresholds: a new, quick, and reproducible method for the assessment of peripheral neuropathy in diabetes mellitus. Diabetologia 32:724-728

21. Weseley SA, Liebowitz B, Katims JJ (1989) Neuropathy of uremia: evaluation by nerve conduction velocity versus neurospecific current perception threshold. Nephron 52:317-322

22. Sakai T, Tomiyasu S, Yamada H, Sumikawa K (2006) Evaluation of allodynia and pain associated with postherpetic neuralgia using current perception threshold testing. Clin J Pain 22:359-362

23. Ro LS, Chen ST, Tang LM, Hsu WC, Chang HS, Huang CC (1999) Current perception threshold testing in Fabry's disease. Muscle Nerve 22:1531-1537

24. Magni G, Caldieron C, Rigatti-Luchini S, Merskey H (1990) Chronic musculoskeletal pain and depressive symptoms in the 
general population: an analysis of the 1st national health and nutrition examination survey data. Pain 43:299-307

25. Magni G, Marchetti M, Moreschi C, Merskey H, Luchini SR (1993) Chronic musculoskeletal pain and depressive symptoms in the National Health and Nutrition Examination. I. Epidemiologic follow-up study. Pain 53:163-168

26. Merskey H, Bogduk N (1994) Classification of chronic pain. IASP Press, Seattle

27. Kimura J, Powers JM, Van Allen MW (1969) Reflex response of orbicularis oculi muscle to supraorbital nerve stimulation. Study in normal subjects and in peripheral facial paresis. Arch Neurol 21:193-199

28. Watson JC (2007) From paroxysmal to chronic pain in trigeminal neuralgia: implications of central sensitization. Neurology 69:817-818

29. Shahani B (1970) The human blink reflex. J Neurol Neurosurg Psychiatry 33:792-800

30. Hilton DA, Love S, Gradidge T, Coakham HB (1994) Pathological findings associated with trigeminal neuralgia caused by vascular compression. Neurosurgery 35:299-303 discussion 303

31. Devor M, Govrin-Lippmann R, Rappaport ZH (2002) Mechanism of trigeminal neuralgia: an ultrastructural analysis of trigeminal root specimens obtained during microvascular decompression surgery. J Neurosurg 96:532-543

32. Marinkovic S, Todorovic V, Gibo H et al (2007) The trigeminal vasculature pathology in patients with neuralgia. Headache 47:1334-1339

33. Rappaport ZH, Govrin-Lippmann R, Devor M (1997) An electron-microscopic analysis of biopsy samples of the trigeminal root taken during microvascular decompressive surgery. Stereotact Funct Neurosurg 68:182-186
34. Basbaum AI, Gautron M, Jazat F, Mayes M, Guilbaud G (1991) The spectrum of fiber loss in a model of neuropathic pain in the rat: an electron microscopic study. Pain 47:359-367

35. Ebenezer GJ, Hauer P, Gibbons C, McArthur JC, Polydefkis M (2007) Assessment of epidermal nerve fibers: a new diagnostic and predictive tool for peripheral neuropathies. J Neuropathol Exp Neurol 66:1059-1073

36. Sommer C, Lauria G (2007) Skin biopsy in the management of peripheral neuropathy. Lancet Neurol 6:632-642

37. Battista AF, Alban E (1983) Effect of graded ligature compression on nerve conduction. Exp Neurol 80:186-194

38. Daemen MA, Kurvers HA, Kitslaar PJ, Slaaf DW, Bullens PH, Van den Wildenberg FA (1998) Neurogenic inflammation in an animal model of neuropathic pain. Neurol Res 20:41-45

39. Ali Z, Ringkamp M, Hartke TV et al (1999) Uninjured C-fiber nociceptors develop spontaneous activity and alpha-adrenergic sensitivity following L6 spinal nerve ligation in monkey. J Neurophysiol 81:455-466

40. Woolf CJ, Mannion RJ (1999) Neuropathic pain: aetiology, symptoms, mechanisms, and management. Lancet 353:19591964

41. Sorensen L, Molyneaux L, Yue DK (2006) The relationship among pain, sensory loss, and small nerve fibers in diabetes. Diabetes Care 29:883-887

42. Herrmann DN, McDermott MP, Sowden JE et al (2006) Is skin biopsy a predictor of transition to symptomatic HIV neuropathy? A longitudinal study. Neurology 66:857-861

43. Woolf CJ (1983) Evidence for a central component of post-injury pain hypersensitivity. Nature 306:686-688 